\title{
FutureJournal
}

\section{A Questão Cultural e sua Influência na Gestão de Equipes de Projetos Globais}

\author{
Nadia Lima \\ curso não identificado pela Universidade Presbiteriana Mackenzie, Brasil \\ nadialima@hotmail.com \\ Leandro Alves Patah \\ Doutor em Engenharia de Produção pela Universidade de São Paulo (USP), Brasil \\ leandro.patah@uol.com.br
}

\section{RESUMO}

As interações entre o gerente de projetos e os membros da equipe podem ser afetadas por diversas variáveis, como a cultura, o estilo de liderança do gerente de projetos e a complexidade das tarefas desenvolvidas. Com foco nesse contexto, o objetivo geral neste artigo é investigar e descrever como a questão cultural pode influenciar a gestão de equipes de projetos globais. Trata-se de um estudo qualitativo e descritivo, realizado em uma empresa multinacional de grande porte do setor automobilístico. Os resultados obtidos nesta pesquisa demonstram que a questão cultural pode influenciar de forma tanto positiva quanto negativa a gestão de equipes de projetos globais e que os gerentes desse tipo de projetos têm de lidar com vários desafios gerenciais que exigem a adoção de determinadas formas de lidar com os impactos da cultura na gestão de suas equipes para minimizar os possíveis problemas existentes nesse contexto.

PALAVRAS-ChAVE: Cultura. Gestão de equipes de projetos. Projetos globais. 


\section{Cultural Issue and its Influence in the Management of Global Project}

\section{Teams}

\section{ABSTRACT}

The interactions between the project manager and team members may be affected by several variables, such as culture, leadership style of the project manager, and the complexity of the developed tasks. Focused in this context, the objective of this paper is to investigate and describe how the culture issue can affect the management of global project teams. It is a qualitative, descriptive study conducted in a large multinational company in the automotive sector. The results of this research show that cultural issues can influence both positively and negatively the management of project global teams and the managers of these projects have to deal with several management challenges that require the adoption of certain ways of dealing with culture impacts in managing their teams to minimize potential problems in this context.

KEY-WORDS: Culture. Project team management. Global projects. 


\section{INTRODUÇÃO}

Com a maior diversidade da força de trabalho atual, as interações multiculturais econômicas, políticas e sociais estão cada vez mais intensas. Nas situações relacionadas ao trabalho, essas interações ocorrem frequentemente, em variadas circunstâncias. Em um ambiente cultural não familiar, pode haver dificuldades com relação a idioma e comunicação, religião, política e, consequentemente, muitos desentendimentos e conflitos podem ocorrer devido, em parte, à incerteza causada pela diversidade cultural (Lin, Chen \& Song, 2012).

A cultura determina, em grande medida, a maneira como as pessoas e as organizações operam no dia a dia, e vários problemas enfrentados pelas empresas ocorrem, muitas vezes, devido aos conflitos causados por culturas distintas (Rodrigues, 2010). Por isso, a diversidade encontrada naturalmente entre os colaboradores de uma empresa requer maior atenção ao gerenciamento das diferenças para que os relacionamentos sejam conduzidos com habilidade e sensibilidade e para que o líder tenha um relacionamento adequado com seus liderados (Russo, Ruiz \& Cunha, 2005).

Dentre as habilidades exigidas de um gerente de projetos, está a capacidade de compreensão da cultura das pessoas dentro de sua equipe (Kerzner, 2000). Ter o conhecimento adequado a respeito da cultura dos membros da equipe é um desafio com o qual quase todos os gerentes de projeto têm de lidar diariamente. Geralmente, os gerentes de projeto não são educados para a diversidade cultural ou não estão em sintonia com ela, e a falta desse conhecimento traz problemas para a gestão em razão dos mais variados tipos de mal-entendido, por isso essa questão deve ser vista pelos gestores de projetos como positiva e um desafio a ser enfrentado (Obikunle, 2002). A conscientização sobre as diferentes culturas ajuda esses gerentes a desenvolver e gerenciar suas equipes de forma mais eficaz. É importante destacar que ter esse conhecimento é particularmente importante nos casos de equipes de projetos que enfrentam problemas de indiferença, hostilidade ou pressão sobre a missão da equipe (Elmes \& Wilemon, 1988). 
A compreensão do impacto das influências culturais é essencial em projetos globais, e a competência multicultural torna-se um fator crítico para o gerente de projetos (PMI, 2013). O gerente de projetos globais precisa reconhecer que a diversidade cultural pode influenciar, negativa ou positivamente, o sucesso do projeto. Ademais, é fundamental ao gerente de projetos identificar as potenciais vantagens e desvantagens competitivas trazidas à equipe pelos profissionais de diferentes culturas, pois, independentemente da orientação cultural, ela pode ser valiosa se o gerente souber administrá-la (Rodrigues, 2010).

Conforme Jetu, Riedl e Roithmayr (2010), ainda existem significantes lacunas no entendimento de como padrões culturais influenciam o comportamento dos membros da equipe. Por isso, estudar a questão cultural e sua influência na gestão de equipes de projetos globais torna-se relevante tanto para a área de gestão de pessoas quanto para a de projetos, já que a coordenação de equipes de projetos globais apresenta uma série de desafios, que começam quando indivíduos de diferentes organizações, diferentes países e diferentes sistemas de valores devem compartilhar autoridade, responsabilidade e tomada de decisão (Shore \& Cross, 2005). Ademais, a cultura é um fator que ajuda a explicar as diferenças, mas pode não só ter uma contribuição benéfica, por exemplo, no aumento da criatividade proporcionada por esses ambientes heterogêneos, como também ser causa de insucesso nesses tipos de projetos (Rodrigues, 2010).

À vista disso, este estudo destina-se a contribuir para uma melhor compreensão da questão cultural no ambiente de gestão de projetos e de como seus gerentes podem lidar com o impacto de diferentes culturas na gestão de equipes de projetos globais. Já que projetos globais possuem características específicas que os tornam mais difíceis de gerenciar, como políticas estrangeiras e questões culturais, além disso, têm um maior impacto na organização se bem sucedidos ou não. Esses projetos dependem de resultados maiores quando comparados a projetos locais e oferecem mais riscos e complexidade, mas também têm mais potenciais benefícios ao mesmo tempo em que são mais custosos (Battistuzzo \& Piscopo, 2015).

Assim, nesta pesquisa, busca-se responder a seguinte questão de pesquisa: Como a questão cultural pode influenciar a gestão de equipes de 
projetos globais de empresas multinacionais? O objetivo geral deste estudo é investigar e descrever como a diversidade cultural dos envolvidos em um projeto pode influenciar a gestão das equipes de projetos globais em empresas multinacionais.

Para responder a questão de pesquisa deste estudo e o objetivo geral proposto, a metodologia utilizada foi a realização de uma pesquisa bibliográfica e de um estudo de natureza descritiva e qualitativa, cuja coleta de dados foi feita por meio de entrevistas semiestruturadas, com apoio de um roteiro de questões e aplicação de um questionário para o levantamento do grau de concordância dos profissionais entrevistados com as proposições estabelecidas neste estudo. A técnica de avaliação dos dados foi a análise do conteúdo das entrevistas realizadas.

Neste estudo há limitações em relação à amostra que gerou resultados apenas do ponto de vista dos gerentes dos projetos selecionados e não de suas equipes, o que reflete resultados cujas opiniões, percepções e perspectivas são baseadas nas experiências e na visão desses profissionais. Também é importante esclarecer que os resultados deste estudo não podem ser generalizados em razão da própria natureza do tema e do procedimento metodológico adotado. Porém, como contribuição deste estudo, os resultados apresentados, que são considerações baseadas nas experiências de profissionais envolvidos em projetos globais, podem ser discutidos ou adotados por outras organizações e profissionais da área de gestão de projetos. Tais resultados podem ainda ser utilizados em estudos futuros relacionados à cultura no ambiente de gestão de projetos globais.

\section{REFERENCIAL TEÓRICO}

\subsection{CULTURA: CONCEITOS E ABORDAGENS}

O conceito de cultura é essencial e muito utilizado porque atende a diversas necessidades e interesses da sociedade e de pesquisadores. A cultura envolve estabilidade, salienta demonstrações conceituais e serve como fator de união para levar os membros do grupo em direção ao consenso (Pires \& Macedo, 2006). 
Diversas definições de cultura podem ser encontradas na literatura (Jetu et al., 2010). Stewart (2006), por exemplo, define cultura como a soma total das crenças, normas, técnicas, instituições e artefatos que caracterizam populações humanas. Para Zein (2012) a cultura é uma coleção de valores, normas, crenças, costumes, instituições e formas de expressão que refletem os pensamentos, sentimentos, ações e interesses das pessoas.

A cultura também pode ser caracterizada como um conjunto compartilhado de atributos de algum grupo, já que esse grupo organiza sua vida, seu ambiente e suas soluções para as questões sociais em conjunto (Obikunle, 2002). Segundo Pires e Macedo (2006), abordar o tema cultura significa tratar a questão de adaptação do indivíduo à realidade do grupo no qual está inserido. A cultura, focada na construção do significado social e em regras e normas, possibilita que um grupo se fortaleça ou se desagregue.

A cultura reflete os valores e as crenças que os membros de um grupo compartilham. Tais valores são expressos por meio de símbolos, como mitos, rituais, histórias, lendas e uma linguagem especializada, influenciando os indivíduos de determinada cultura quanto à forma de pensar, agir e tomar decisões (Pires \& Macedo, 2006).

\subsubsection{Cultura organizacional}

Cultura organizacional é uma das mais influentes dimensões do ambiente de trabalho e, consequentemente, a principal força de direção de um negócio. Ela reflete-se na maneira como as tarefas são realizadas, as metas são alcançadas e as pessoas são direcionadas para o atingimento de metas. A cultura afeta a tomada de decisão, a forma de pensar, sentir e responder às oportunidades e às ameaças. A cultura está enraizada nas pessoas e inconscientemente influencia seus comportamentos e afeta seu desempenho e vice-versa (Stare, 2011).

A cultura é um fenômeno dinâmico que permeia os indivíduos em todos os momentos e é constantemente promulgada e criada pelas interações dos indivíduos e moldada pelo comportamento da liderança, 
como um conjunto de estruturas, rotinas, regras e normas que orientam e restringem o comportamento. Na organização e até mesmo nos grupos dentro da organização, pode-se observar como a cultura é criada, incorporada, evolui e é manipulada, e, ao mesmo tempo, como a cultura constrange, estabiliza e fornece estrutura e significado aos membros do grupo (Schein, 2004).

A cultura e o estilo da organização são fatores ambientais da empresa que podem influenciar a forma como os projetos são conduzidos. A cultura organizacional é estruturada com base nas experiências comuns dos membros da organização, e a maioria das empresas desenvolve culturas únicas no decorrer do tempo através da prática e do uso comum. Algumas dessas experiências incluem visões compartilhadas, missão, valores, crenças e expectativas; regulamentos, políticas, métodos e procedimentos; sistemas de motivação e recompensa; tolerância a riscos; visão das relações de liderança, hierarquia e autoridade; código de conduta, ética e hora de trabalho, e ambientes operacionais (PMI, 2013).

No âmbito do gerenciamento de projetos, a cultura organizacional pode influenciar como os departamentos interagem e se apoiam mutuamente na busca de objetivos do projeto. Também influencia o nível de comprometimento dos empregados no alcance dos objetivos do projeto, a fim de equilibrá-los com outros objetivos existentes e potencialmente concorrentes. A cultura organizacional ainda influencia o processo de planejamento do projeto assim como a forma de trabalho é estimada ou como os recursos dos projetos são designados. Por fim, a cultura afeta a maneira como os gerentes avaliam o desempenho da equipe de projetos e como eles entendem o resultado do projeto (Stare, 2011).

\subsubsection{Diversidade cultural}

Apesar do contexto cultural diversificado em que estão inseridas as empresas brasileiras, a questão da diversidade cultural é um tema recente, assim como o interesse das empresas na gestão da diversidade cultural, que surgiu nos anos 1990. O tema da diversidade cultural nas organizações 
também tem sido considerado como um fator de vantagem estratégica para as empresas (Hanashiro \& Carvalho, 2005).

A diversidade cultural consiste em um conjunto de pessoas com identidades diferentes interagindo no mesmo sistema social. Esse conceito está relacionado ao reconhecimento e ao respeito à individualidade dos empregados, e gerenciar a diversidade implica o desenvolvimento das competências necessárias ao crescimento e ao sucesso do negócio (Fleury, 2000). Por isso, administrar a diversidade cultural requer uma abordagem holística para desenvolver um ambiente organizacional que permita a todos desenvolver plenamente seu potencial no alcance dos objetivos da empresa (Thomas, 1996).

As diferenças culturais interagem com um conjunto de fatores individuais, grupais e organizacionais a fim de estabelecer o impacto da diversidade no desempenho individual e organizacional. O desempenho individual é dividido em variáveis de resposta afetiva, como satisfação, identificação organizacional e envolvimento no trabalho, e em variáveis de desempenho (performance, mobilidade no cargo e compensação). Já os resultados organizacionais podem influenciar a qualidade do atendimento, de turnover, de qualidade no trabalho e de lucratividade (Cox, 1994).

No contexto de gerenciamento de projetos, a diversidade cultural é um tema no qual os gerentes de projetos devem focar sua atenção a fim de serem bem sucedidos (Obikunle, 2002). Nesse ambiente, a diversidade cultural pode ser determinada por diversos fatores. Segundo o modelo desenvolvido por Miliken e Martins (1996), a diversidade apresenta diferentes dimensões e tem impactos diferentes no curto e no longo prazo na produtividade de grupos heterogêneos. Miliken e Martins (1996) dividem os impactos da diversidade organizacional sobre os indivíduos em duas dimensões temporais de curto e longo prazo e por tipos de impacto: os impactos afetivos, relacionados à identificação e à satisfação da pessoa com o grupo; e os cognitivos, considerados como a habilidade do indivíduo em processar a informação, perceber e interpretar estímulos, além de tomar decisões. Na Figura 1, apresentam-se os impactos da diversidade cultural sobre os indivíduos. 


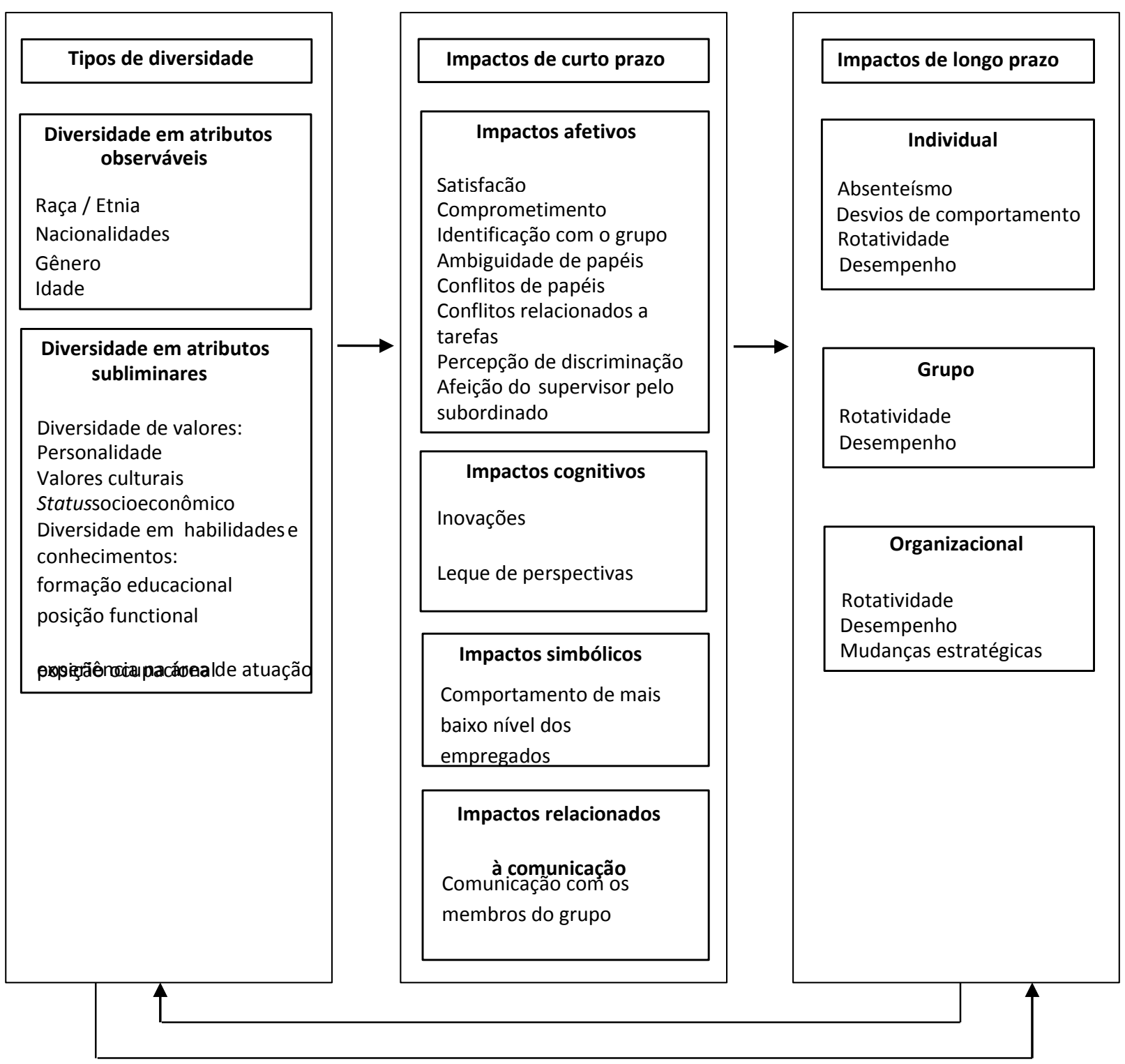

\section{Figura 1: Impactos da diversidade cultural sobre os indivíduos}

Fonte adaptado de: Miliken e Martins (1996)

Os impactos da diversidade cultural podem ser tanto positivos como negativos, já que, além de algumas vantagens, também podem gerar problemas motivacionais, conflitos, rotatividade e dificuldades de comunicação, afetando, consequentemente, a produtividade (Miliken \& Martins, 1996).

Segundo Cox (1994), a diversidade cultural presente nas organizações pode causar impactos tanto em termos tanto da eficácia organizacional como individual. E o ambiente organizacional é importante para determinar se esse impacto será positivo ou negativo. 


\subsection{PROJETOS GLOBAIS}

Cada vez mais os projetos têm sido desenvolvidos além das fronteiras nacionais e isso tem trazido uma série de desafios gerenciais, já que pessoas de diferentes valores, organizações e países têm de compartilhar autoridade, responsabilidade e tomada de decisões (Shore \& Cross, 2005). Um projeto global é definido como um projeto multinacional e temporário em que diversos integrantes procuram otimizar resultados por meio da combinação de recursos de diversas localidades, organizações e estilos organizacionais (Orr, Scott, Levitt, Artto \& Kujala, 2011).

Os projetos globais têm características específicas que os tornam mais difíceis de gerenciar, como políticas estrangeiras e questões culturais, além disso, têm maior impacto na organização se bem sucedidos ou não. Esses projetos dependem de resultados maiores quando comparados a outros locais e oferecem mais riscos e complexidade, mas também têm mais potenciais benefícios ao mesmo tempo em que são mais custosos (Battistuzzo \& Piscopo, 2015).

Considerando que projetos globais terão de lidar com culturas diversas, o papel da cultura assume maior importância em seu desempenho. Como todos os membros da equipe levam sua cultura para o projeto, o gerente de projetos precisa estar ciente e entender como a cultura poderá impactar o projeto global (Anantatmula \& Thomas, 2010).

\subsubsection{Gestão de equipes de projetos globais}

O trabalho em equipe eficaz é fundamental para o sucesso do projeto, mas também é difícil de gerenciar (Thanhaim, 2012). O gerenciamento das equipes de projetos é complexo por duas razões: primeiro porque a equipe de projetos é extremamente dinâmica e os membros do grupo estão em constante mudança; segundo porque, possivelmente, somente o gerente do projeto e alguns membros da alta gerência compreendem a equipe do projeto como uma entidade única (Patah \& Carvalho, 2002).

Atualmente, em um ambiente complexo, multinacional e tecnologicamente intrincado, a equipe do projeto, pode ser definida como 
um conjunto de indivíduos, selecionados por suas habilidades e qualidades específicas. É um grupo que possui necessidades, origens e experiências diferentes que devem ser habilmente focadas a fim de transformar esse grupo de trabalho em uma equipe integrada e unificada (Thanhaim, 2012).

De acordo com Punzo (1996), o gerente de projetos deve abordar a diferença cultural entre os membros da equipe, para facilitar uma comunicação produtiva, as relações interpessoais, a resolução de problemas, o trabalho em equipe e a sinergia requerida para conceituar, planejar, implementar e avaliar projetos com sucesso.

O gerente do projeto deve adaptar seu estilo de liderança às características dos membros da equipe, compreendendo como seus membros se comportam em relação à hierarquia, ao coletivismo e à incerteza nos projetos. Além disso, o gerente de projetos deve definir canais de comunicação efetivos a fim de evitar problemas de entendimento quanto a prazos, qualidade e custos (Rodrigues, 2010).

Para desenvolver equipes eficientes, os gerentes de projetos devem ser flexíveis e estar dispostos a apoiar o time. Eles devem querer ajudar os membros da equipe a trabalharem juntos, entenderem suas diferenças culturais e facilitar a comunicação. Eles ainda devem possuir uma mentalidade global para responder prontamente e de forma criativa aos desafios que podem surgir (Segil, 1999).

Em projetos globais, a equipe é composta de pessoas de diferentes países, trabalhando em diferentes culturas, unidades de negócios e funções e com conhecimento específico para resolver uma atividade estratégica comum (Anantatmula \& Thomas, 2010). Equipes de projetos globais vivenciam o desafio de conseguir um grupo diversificado de indivíduos de diferentes áreas funcionais para trabalharem juntos efetivamente em um período determinado de tempo a fim de alcançar objetivos específicos do projeto (Barczak \& McDonough, 2003).

A cultura da equipe de projetos globais apresenta alto grau de diversidade de valores, crenças, estilo de liderança, entre outras diferenças, e consequentemente requer um estilo de gestão focado na integração de seus membros, na cooperação, no compartilhamento do poder, no comprometimento, dentre outros fatores (Rodrigues, 2010). 
Equipes de projetos globais devem construir a confiança entre os membros da equipe, cumprir horários e aderir às diretrizes orçamentárias. Também devem enfrentar o desafio da distância física, da diversidade cultural, das barreiras de idiomas e das diferenças de infraestrutura tecnológica (Barczak \& McDonough, 2003).

As distâncias geográficas e temporais das equipes em projetos globais podem causar maiores dificuldades do que em projetos tradicionais (Lee-Kelley \& Sankey, 2008). As barreiras de comunicação são ampliadas em equipes globais virtuais pela distância, pela diversidade cultural, pelo idioma, pela diferença de fuso horário e pela confiança entre os membros da equipe (Anantatmula \& Thomas, 2010).

Faz-se importante ressaltar que a multiculturalidade em equipes de projetos globais também traz uma série de vantagens, como variedade de perspectivas, de habilidades e de características pessoais que contribuem para uma organização. A multiculturalidade pode ser muito valiosa, já que equipes multiculturais costumam criar uma cultura interna, que direciona os esforços e aumenta a coesão da equipe, facilitando a comunicação e o bom desempenho (Rodrigues, 2010).

\section{MÉTOdO E TÉCNICAS DE PESQUISA}

Este estudo caracteriza-se por uma pesquisa qualitativa e descritiva para examinar como a questão cultural pode influenciar a gestão de equipes de projetos globais em empresas multinacionais. A unidade de análise desta pesquisa são dois projetos globais de uma empresa multinacional de grande porte do ramo automobilístico.

Para a coleta de dados, inicialmente foram elaboradas proposições fundamentadas no referencial teórico deste estudo e, com base nessas proposições, foram desenvolvidos tanto um roteiro de entrevista com questões semiabertas, como um questionário para o levantamento do nível de concordância dos entrevistados com as afirmações apresentadas.

As entrevistas foram realizadas pessoalmente com cada um dos gerentes, e utilizaram-se as questões do roteiro de entrevista, a fim de verificar como a questão cultural pode influenciar a gestão de equipes de 
projetos globais. Ao término da entrevista, foi entregue aos respondentes o questionário para indicarem o grau de concordância com as afirmações apresentadas, com o objetivo de averiguar se os relatos dos profissionais entrevistados estavam em acordo com as proposições descritas no estudo. Além das entrevistas e dos questionários, foram coletadas informações sobre cada projeto.

Para o estudo dessas diferentes fontes de dados, o método utilizado foi a análise de conteúdo das entrevistas, posteriormente foram avaliadas as respostas ao questionário comparando-as entre si e também com as proposições estabelecidas, e em paralelo, foi feita a análise das informações disponibilizadas pelos gestores sobre cada projeto. Os dados analisados foram separados em categorias (influências positivas e negativas e formas de lidar com os impactos da cultura) e descritos no item 4 - interpretação e apresentação dos resultados.

\subsection{PROPOSIÇÕES DA PESQUISA}

A partir da revisão bibliográfica foram formuladas proposições, conforme Quadro 1, para elaboração das questões da pesquisa de campo.

\begin{tabular}{|c|c|c|}
\hline Proposições & Autores & Questões \\
\hline $\begin{array}{l}\text { A. As diferenças culturais interagem com um } \\
\text { conjunto de fatores individuais, grupais e } \\
\text { organizacionais a fim de estabelecer o } \\
\text { impacto da diversidade no desempenho } \\
\text { individual e organizacional. }\end{array}$ & Cox (1994) & $\begin{array}{l}\text { 1. Em sua opinião, como as } \\
\text { diferenças culturais } \\
\text { existentes nos projetos } \\
\text { globais podem impactar o } \\
\text { desempenho individual e } \\
\text { organizacional? }\end{array}$ \\
\hline $\begin{array}{l}\text { B. A cultura é tanto um fenômeno dinâmico } \\
\text { que nos cerca em todos os momentos, } \\
\text { sendo constantemente promulgada e } \\
\text { criada por nossas interações com os } \\
\text { outros e moldada pelo comportamento da } \\
\text { liderança, como um conjunto de } \\
\text { estruturas, rotinas, regras e normas que } \\
\text { orientam e restringem o comportamento. }\end{array}$ & Schein (2004) & $\begin{array}{l}\text { 2. Com base em sua } \\
\text { experiência profissional, } \\
\text { como a cultura pode impactar } \\
\text { a relação do gerente de } \\
\text { projetos globais e sua } \\
\text { equipe? }\end{array}$ \\
\hline
\end{tabular}




\begin{tabular}{|c|c|c|}
\hline Proposições & Autores & Questões \\
\hline $\begin{array}{l}\text { C. Ter o conhecimento adequado a respeito } \\
\text { da cultura dos membros da equipe é um } \\
\text { desafio com o qual quase todos os } \\
\text { gerentes de projeto têm de lidar } \\
\text { diariamente. Geralmente, os gerentes de } \\
\text { projeto não são educados ou não estão } \\
\text { em sintonia com a diversidade cultural, e } \\
\text { a falta desse conhecimento traz problemas } \\
\text { para a gestão em razão dos mais variados } \\
\text { tipos de mal-entendido. }\end{array}$ & Obikunle (2002) & $\begin{array}{l}\text { 3. Com base em sua } \\
\text { experiência, quais problemas } \\
\text { podem ocorrer pela falta do } \\
\text { conhecimento adequado do } \\
\text { gerente de projetos a } \\
\text { respeito da cultura dos } \\
\text { membros da equipe? }\end{array}$ \\
\hline $\begin{array}{l}\text { D. É fundamental, ao gerente de projetos, } \\
\text { identificar as potenciais vantagens e } \\
\text { desvantagens competitivas trazidas à } \\
\text { equipe pelos profissionais de diferentes } \\
\text { culturas, pois, independentemente da } \\
\text { orientação cultural, ela pode ser valiosa se } \\
\text { o gerente souber administrá-la. }\end{array}$ & $\begin{array}{c}\text { Rodrigues } \\
\text { (2010); Cox } \\
\text { (1994); Miliken } \\
\text { e Martins } \\
(1996)\end{array}$ & 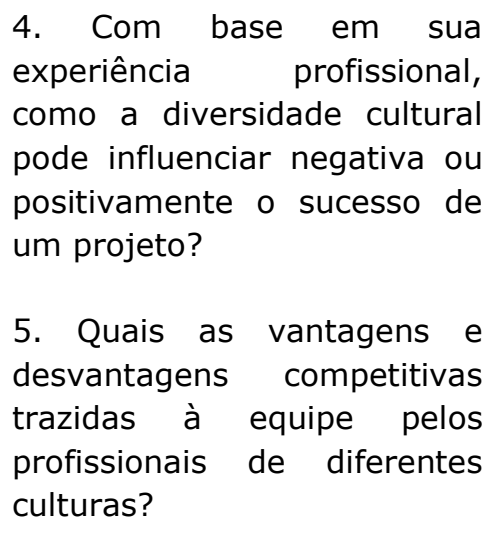 \\
\hline $\begin{array}{l}\text { E. A cultura afeta a forma como os gerentes } \\
\text { avaliam o desempenho da equipe de } \\
\text { projetos e como eles veem o resultado do } \\
\text { projeto. }\end{array}$ & Stare (2011) & $\begin{array}{l}\text { 6. De acordo com o seu } \\
\text { ponto de vista, como a } \\
\text { cultura pode influenciar a } \\
\text { maneira como os gerentes } \\
\text { avaliam o desempenho da } \\
\text { equipe de projetos? }\end{array}$ \\
\hline $\begin{array}{l}\text { F. Os projetos são cada vez mais } \\
\text { desenvolvidos além das fronteiras } \\
\text { nacionais, e isso tem trazido uma série de } \\
\text { desafios gerenciais, já que pessoas de } \\
\text { diferentes organizações e países, e com } \\
\text { diferentes valores, têm de compartilhar } \\
\text { autoridade, responsabilidade e tomada de } \\
\text { decisões. }\end{array}$ & $\begin{array}{c}\text { Shore e Cross } \\
(2005)\end{array}$ & $\begin{array}{l}\text { 7. Com base em sua } \\
\text { experiência, quais os desafios } \\
\text { gerenciais que os projetos } \\
\text { globais podem apresentar, já } \\
\text { que pessoas de diferentes } \\
\text { organizações e países, e com } \\
\text { diferentes culturas e valores } \\
\text { têm de compartilhar } \\
\text { autoridade, responsabilidade } \\
\text { e tomada de decisões? }\end{array}$ \\
\hline
\end{tabular}




\begin{tabular}{|c|c|c|}
\hline Proposições & Autores & Questões \\
\hline $\begin{array}{l}\text { G. Faz-se importante ressaltar que, a } \\
\text { multiculturalidade em equipes de projetos } \\
\text { globais também traz uma série de } \\
\text { vantagens, como variedade de } \\
\text { perspectivas, de habilidades e de } \\
\text { características pessoais que contribuem } \\
\text { para uma organização. }\end{array}$ & $\begin{array}{l}\text { Rodrigues } \\
(2010)\end{array}$ & $\begin{array}{l}\text { 8. A multiculturalidade em } \\
\text { equipes de projetos globais } \\
\text { pode trazer uma série de } \\
\text { vantagens. Em sua opinaão, } \\
\text { quais são essas vantagens? }\end{array}$ \\
\hline Proposições & Autores & Questões \\
\hline $\begin{array}{l}\text { H. O gerente de projetos deve abordar a } \\
\text { diferença cultural entre os membros da } \\
\text { equipe, para facilitar uma comunicação } \\
\text { produtiva, as relações interpessoais, a } \\
\text { resolução de problemas, o trabalho em } \\
\text { equipe e a sinergia requerida para } \\
\text { conceituar, planejar, implementar e } \\
\text { avaliar projetos com sucesso. }\end{array}$ & Punzo (1996) & $\begin{array}{l}\text { 10. Por que o gerente de } \\
\text { projetos deve abordar a } \\
\text { diferença cultural entre os } \\
\text { membros da equipe? }\end{array}$ \\
\hline
\end{tabular}

\section{Quadro 1: Proposições e questões de pesquisa}

\subsection{UNIDADE DE ANÁLISE}

A unidade de análise desta pesquisa são dois projetos globais, denominados Projeto A e Projeto B. O Projeto A foi desenvolvido para adequação tecnológica de veículos nacionais e importados às novas exigências de controle de emissão de poluentes. Coordenado por um gerente de projetos estratégicos, este projeto envolvia uma equipe multidisciplinar de diversas áreas funcionais, como área financeira, engenharia, qualidade, logística, compras, marketing e outros. Já o Projeto B tratava do desenvolvimento de uma solução para melhoria e maior eficiência da mobilidade urbana. Coordenado por um gerente da área de vendas para o mercado externo, envolvia uma equipe multidisciplinar das áreas de vendas, financeira e marketing de produto.

\section{INTERPRETAÇÃO E APRESENTAÇÃO DOS RESULTADOS}

Com base nas informações fornecidas pelos profissionais entrevistados nesta pesquisa e na revisão bibliográfica realizada, nos 
resultados obtidos demonstra-se que a diversidade cultural existente entre os envolvidos em um projeto global e também a cultura da própria empresa são fatores críticos para o desempenho do projeto e dos membros da equipe de projetos, e que as diferentes culturas podem trazer ao ambiente de projetos tanto influências negativas quanto positivas.

Em decorrência disso, é necessário que os gerentes de projetos com equipes multiculturais compreendam e saibam como administrar essas diferenças, a fim de promover uma maior integração e sinergia entre todos os envolvidos no projeto. Na Figura 2, apresentam-se tanto as influências positivas quanto as negativas que a cultura pode trazer ao ambiente de projetos e que foram mencionadas pelos profissionais entrevistados, e ainda um conjunto de ações que os gerentes de projetos globais podem adotar para lidar com os diferentes aspectos culturais existentes nesse tipo de ambiente.

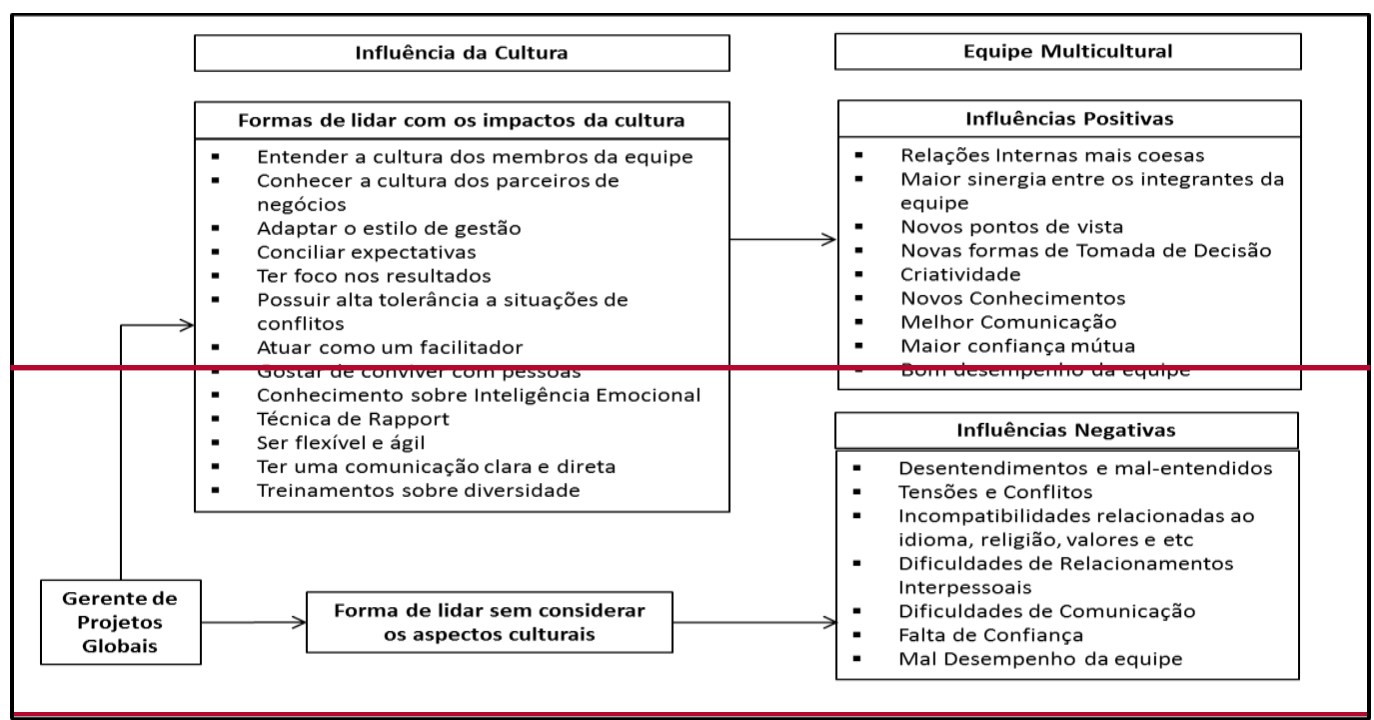

Figura 2: A questão cultural e sua influência na gestão de equipes de projetos globais

Fonte: resultado da interpretação dos dados obtidos

Com base nas informações mencionadas pelos profissionais entrevistados, a categoria denominada como influências negativas, que podem comprometer o desempenho da equipe e do projeto, diz respeito à falta do conhecimento adequado do gerente de projetos quanto às diferentes culturas dos membros da equipe, fato que pode acarretar desentendimentos, dificuldades de relacionamento e comunicação, falta de 
confiança, tensões, conflitos diversos e incompatibilidades em relação a religiosidade, costumes, valores, alimentação e idioma, principalmente nas equipes que se encontram distantes geograficamente e com as quais se mantém contato remoto. Todos esses fatores citados estão de acordo com a afirmação de Lin et al. (2012), que esclarecem que nas interações multiculturais relacionadas ao trabalho pode haver dificuldades com relação a idioma e comunicação, religião, política e muitos desentendimentos e conflitos causados pela diversidade cultural. Esses fatores também corroboram a proposição C desta pesquisa em que Obikunle (2002) explica que, ter o conhecimento adequado a respeito da cultura dos membros da equipe é um desafio com o qual quase todos os gerentes de projeto têm de lidar diariamente, pois, geralmente, esses profissionais não são educados ou não estão em sintonia com a diversidade cultural, e a falta desse conhecimento traz problemas para a gestão em razão dos mais variados tipos de mal-entendido.

Já a categoria mencionada como influências positivas, que também podem ter influência no desempenho da equipe e do projeto, foi considerada pelos respondentes da pesquisa como os aspectos que podem trazer vantagens para o projeto e para a equipe em razão do ambiente heterogêneo de projetos globais, que propicia novas perspectivas e pontos de vista, diferentes formas de tomada de decisão, maior criatividade e diferentes níveis de experiência e conhecimentos. Todos esses aspectos validam a proposição G desta pesquisa em que Rodrigues (2010) afirma que a multiculturalidade em equipes de projetos globais também traz uma série de vantagens, como variedade de perspectivas, de habilidades e de características pessoais que contribuem para uma organização.

Quanto à categoria classificada como formas de lidar com os impactos da cultura, os profissionais entrevistados relataram que, para gerir de forma adequada a diversidade de uma equipe multicultural, o gerente de projetos precisa ser capaz de entender a cultura dos membros de sua equipe e das pessoas ligadas ao projeto com as quais ele se relaciona, para que possa entender melhor o comportamento de cada envolvido no projeto. Esse resultado está em acordo com a afirmação de Kerzner (2000) que defende que, dentre as habilidades exigidas de um gerente de projetos, 
está a capacidade de compreensão da cultura das pessoas dentro de sua equipe.

Os profissionais entrevistados também mencionaram que é preciso saber adaptar seu estilo de gestão para adequar o modo de solicitar as tarefas do projeto de uma maneira mais assertiva para cada membro do grupo, conciliando as diferentes direções e expectativas das pessoas. Esse relato está de acordo com a proposição B desta pesquisa, em que Schein (2004) esclarece que a cultura é tanto um fenômeno dinâmico que nos cerca em todos os momentos, sendo constantemente promulgada e criada por nossas interações com os outros e moldada pelo comportamento da liderança, como um conjunto de estruturas, rotinas, regras e normas que orientam e restringem o comportamento. Esse mesmo relato dos entrevistados está ainda em concordância com a afirmação de Rodrigues (2010) que explica que o gerente de projetos deve adaptar seu estilo de liderança às características dos membros da equipe, compreendendo como essas pessoas se comportam em relação à hierarquia, ao coletivismo e à incerteza nos projetos.

Vale aqui destacar que, ter foco nos resultados, alta tolerância a situações de conflitos, gostar de conviver com pessoas e atuar como um facilitador foram as habilidades relatadas pelos gerentes entrevistados como as características essenciais que os ajudam na condução de projetos globais.

Além do conhecimento dos valores, das crenças e dos costumes de outras culturas com as quais o gerente de projetos tem de lidar, um dos mecanismos utilizados por esses gerentes de projetos para lidar com as diferenças culturais dos membros da equipe é o conhecimento da técnica de rapport, conceito do ramo da psicologia em que se utiliza uma técnica exclusiva para criar uma ligação de sintonia e empatia com outra pessoa. Os gerentes desenvolvem, dessa forma, tratativas específicas para algumas pessoas e criam maiores vínculos, melhorando a comunicação, as relações internas e a confiança mútua entre os envolvidos no projeto. Essas informações mencionadas pelos respondentes corroboram a afirmação de Thanhaim (2012) que defende que a equipe de projeto é um grupo que possui necessidades, origens e experiências diferentes que devem ser 
habilmente focadas a fim de transformar esse grupo de trabalho em uma equipe integrada e unificada.

Os participantes desta pesquisa esclarecem ainda que o ambiente de projetos globais exige do gerente de projetos ser flexível, ágil na tomada de decisão e, ainda, ter uma comunicação clara e direta, inteligência emocional, sabendo adaptar-se com resiliência à dinâmica e às mudanças constantes nesse ambiente. Esses resultados estão de acordo com a proposição H desta pesquisa, em que Segil (1999) explica que, para desenvolver equipes eficientes, os gerentes de projetos devem ser flexíveis e dispostos a apoiar o time. Devem, ainda, entender as diferenças culturais e ter boa comunicação.

Os profissionais entrevistados também reforçam que é importante indicar para os membros da equipe treinamentos sobre diversidade cultural e abordar esse tema em reuniões com os integrantes da equipe para incentivar que todos saibam da importância da diversidade e compreendam melhor as diferenças, para que possam ser mais eficazes no trabalho com parceiros globais. Todos esses fatores comentados pelos entrevistados estão em concordância com a proposição I desta pesquisa em que Punzo (1996) afirma que o gerente de projetos deve abordar a diferença cultural entre os membros da equipe para facilitar uma comunicação produtiva, as relações interpessoais, a resolução de problemas, o trabalho em equipe e a sinergia requerida para conceituar, planejar, implementar e avaliar projetos com sucesso.

\section{CONSIDERAÇÕES FINAIS}

Neste estudo, o objetivo foi pesquisar e descrever como a questão cultural pode influenciar a gestão de equipes de projetos globais em empresas multinacionais, e ainda como os gerentes de projetos podem lidar com os impactos da cultura na gestão de equipes de projetos globais.

Com base nos resultados desta pesquisa, é possível afirmar que o objetivo geral estabelecido foi cumprido por meio dos resultados obtidos com as questões do roteiro de entrevista, questionário aplicado e revisão bibliográfica efetuada, em que se verificou que as diferentes culturas podem 
influenciar de forma tanto positiva quanto negativa a gestão das equipes de projetos globais, conforme a afirmação de Miliken \& Martins (1996), que esclarecem os impactos da diversidade cultural podem ser tanto positivos como negativos, já que, além de algumas vantagens, também podem gerar problemas motivacionais, conflitos, rotatividade e dificuldades de comunicação afetando, consequentemente, a produtividade.

Por meio dos resultados obtidos, pode-se inferir que as influências positivas da questão cultural na gestão de equipes de projetos globais estão relacionadas à existência de um ambiente heterogêneo e multicultural que possibilita a apresentação de diferentes pontos de vista, novos conhecimentos, maior criatividade e apresentação de diferentes soluções aos problemas que surgem nos projetos, diferentes formas de tomada de decisão e maior incentivo ao respeito às diferentes culturas. Todos esses fatores facilitam uma maior sinergia e favorecem relações mais coesas, possibilitam também uma melhor comunicação e confiança entre os envolvidos no projeto, favorecendo consequentemente um melhor desempenho das equipes, do andamento dos projetos e das organizações.

Já as influências negativas da questão cultural na gestão de equipes de projetos globais estão relacionadas principalmente às dificuldades de relacionamento interpessoal, devido aos diferentes valores, crenças, hábitos e estilos de vida. Essas influências negativas estão também atreladas às dificuldades de comunicação que envolvem a falta de domínio de um segundo idioma, principalmente o inglês, e ainda a falta de clareza, objetividade e transparência nas formas de comunicação, que causam diversos desentendimentos e mal-entendidos, situações tensas e conflitos, que geram a falta de confiança entre os envolvidos no projeto e podem influenciar negativamente o bom desempenho das equipes e dos projetos.

Por meio dos resultados obtidos, pode-se ainda concluir que, devido ao ambiente multicultural e mais complexo que os projetos globais apresentam, seu gerenciamento demanda um gerente de projetos que, além das competências para a gestão de um projeto tradicional, tem de lidar com uma série de desafios que exige um estilo de gerenciamento com certo grau de flexibilidade, capacidade de compreensão e uma mentalidade global, para tratar dos diferentes aspectos culturais que as equipes de 
projetos globais apresentam, de distância geográfica, conflitos e tensões, comunicação, idioma, religião, costumes, valores e confiança mútua. Esses profissionais precisam, ainda, adotar formas de lidar com os impactos da cultura na gestão de suas equipes para minimizar os possíveis problemas existentes nesse contexto.

Por fim, com base nas informações obtidas nesta pesquisa, verifica-se ainda que a questão cultural no ambiente de gestão de projetos é um tema que pode ser trabalhado de forma mais intensa e detalhada no ambiente corporativo em que se desenvolvem projetos globais. Além dos esforços dos próprios gerentes de projetos globais, que buscam no dia a dia desenvolver dentro de suas equipes estratégias para lidar com a diversidade cultural existente nesse ambiente de trabalho, as próprias organizações podem oferecer maior suporte aos gestores e equipes de projetos globais possibilitando a realização de treinamentos sobre diversidade e convivência cultural e de atividades extras de integração para os envolvidos no projeto.

\section{REFERÊNCIAS}

Anantatmula, V., \& Thomas, M. (2010). Managing global projects: a structured approach for better performance. Project Management Journal, 41(2), 60-72.

Barczak, G., \& McDonough III, E. F. (2003). Leading global product development teams. Research Technology Management, 46(6), 14-18.

Battistuzzo, F. J., \& Piscopo, M. (2015). Global projects: a bibliometric study of international business journals. Internext, 10(2), 31-45.

Cox, T. (1994). Cultural diversity in organizations: theory, research and practice. San Francisco: Berrett-Koehler.

Elmes, M., \& Wilemon, D. (1988). Organizational culture and project leader effectiveness. Project Management Journal, 19(4), 54-63.

Fleury, M. T. L. (2000). Gerenciando a diversidade cultural: experiências de empresas brasileiras. Revista de Administração de Empresas - RAE, $40(3), 18-25$.

Hanashiro, D. M. M., \& Carvalho, S. G. (2005). Diversidade cultural: panorama atual e reflexões para a realidade brasileira. Revista Eletrônica de Administração - REAd, 11(5), 1-21. 
Jetu, F. T., Riedl, R., \& Roithmayr, F. (2010). Cultural patterns influencing project team behavior in Sub-Saharan Africa: a case estudy in Ethiopia. Project Management Journal, 42(5), 57-77

Kerzner, H. (2000). Applied project management. Best pratices on implementation. New York: John Wiley \& Sons.

Lee-Kelley, L., \& Sankey, T. (2008). Global virtual teams for value creation and project success: a case study. International Journal of Project Management, 26(1), 51-62.

Lin, Y., Chen, A. S., \& Song, Y. (2012). Does your intelligence help to survive in a foreign jungle? The effects of cultural intelligence and emotional intelligence on cross-cultural adjustment. International Journal of Intercultural Relations, 36(4), 541-552.

Miliken, F. J., \& Martins L. L. (1996). Searching for common threads: understanding the multiple effects of diversity in organizational groups. The Academy of Management Review, 21(2), 402-433.

Obikunle, O. (2002). Dealing with cultural diversity in project management: a dilemma in communication. Proceedings of the PMI Annual Seminar \& Symposium, San Antonio, TX, USA.

Orr, R. J., Scott, W. R., Levitt, R. E., Artto, K., \& Kujala, J. (2011). Global projects: distinguishing features, drivers, and challenges. In W. Scott, R. E. Levitt, \& R. J. Orr (Eds.), Global projects institutional and political challenges. New York: Cambridge University Press.

Patah, L. A., \& Carvalho, M. M. D. (2002). Estruturas de gerenciamento de projetos e competências em equipes de projetos. Anais do Encontro Nacional de Engenharia de Produção, Curitiba, PR, Brasil.

Pires, J. C. S., \& Macedo, K. B. (2006). Cultura organizacional em organizações públicas no Brasil. Revista de Administração Pública - RAP, 40(1), 81-105.

Project Management Institute - PMI (2013). A guide to the Project Management Body of Knowledge - PMBOK® Guide (5th ed.). Pennsylvania: PMI.

Punzo, R. A. (1996). Managing cross-cultural values in project teams. Proceedings of the PMI Annual Seminar/Symposium, 27, Boston, MA, USA.

Rodrigues, I. (2010). Cultura e desempenho em equipes de projetos globais: um estudo em empresas multinacionais brasileiras. Tese de 
Doutorado, Faculdade de Economia, Administração e Contabilidade da Universidade de São Paulo: SP.

Russo, R. F. S. M., Ruiz, J. M., \& Cunha, R. P. D. (2005). Liderança e influência nas fases da gestão de projetos. Revista Produção, 15(3), 362375.

Schein, E. H. (2004). Organizational culture and leadership (3rd ed.). San Francisco, California: Jossey-Bass.

Segil, L. (1999). Global work teams: a cultural perspective. PM Network Journal, 13(3), 25-29.

Shore B., \& Cross, B. J. (2005). Exploring the role national culture in the management of large scale international science projects. International Journal of Project Management, 23(1), 55-64.

Stare, A. (2011). The impact of the organizational structure and project organizational culture on project performance in Slovenian enterprises. Management: Journal of Contemporary Management Issues, 16(2), 1-22.

Stewart, J. (2006). Cross culture project management. Proceedings of the PMI Global Congress - EMEA, Seattle, WA, USA.

Thamhain, H. J (2012). The changing role of team leadership in multinational project environments. Revista de Gestão e Projetos, 3(2), 4-38.

Thomas, D., \& Ely R. (1996). Making differences matter: a new paradigm for managing diversity. Harvard Business Review, 74, 1-16.

Zein, O. (2012). Cultural complexities in multinational projects. Proceedings of the PMI Global Congress - EMEA, Marseille, France. 